\title{
Fusion-based Variational Image Dehazing
}

\author{
Adrian Galdran, Javier Vazquez-Corral, David Pardo, and Marcelo Bertalmío
}

\begin{abstract}
We propose a novel image dehazing technique based on the minimization of two energy functionals and a fusion scheme to combine the output of both optimizations. The proposed Fusion-based Variational Image Dehazing (FVID) method is a spatially varying image enhancement process that first minimizes a previously proposed variational formulation that maximizes contrast and saturation on the hazy input. The iterates produced by this minimization are kept, and a second energy that shrinks faster intensity values of well-contrasted regions is minimized, allowing to generate a set of Difference-of-Saturations (DiffSat) maps by observing the shrinking rate. The iterates produced in the first minimization are then fused with these DiffSat maps to produce a haze-free version of the degraded input. The FVID method does not rely on a physical model from which to estimate a depth map, nor it needs a training stage on a database of human-labeled examples. Experimental results on a wide set of hazy images demonstrate that FVID better preserves the image structure on close-by regions that are less affected by fog, and it successfully compares with other current methods in the task of removing haze degradation from far-away regions.

Index Terms-Image Dehazing, Variational Image Processing, Image Fusion, Color Correction, Contrast Enhancement.
\end{abstract}

\section{INTRODUCTION}

Images acquired in outdoor scenarios often suffer from degradation produced by the atmosphere that lies between the observer and the objects in the scene. This phenomenon, known as haze, distorts contrast and color in the image, decreasing the visibility of contents in the scene and reducing visual quality.

The task of removing haze degradation from an image is known as image dehazing, and has recently given rise to an important area of research. A first family of methods rely on inverting a physical model of the degradation, such as Koschmieder's atmospheric scattering model [1]:

$$
\mathrm{I}(x)=\mathrm{t}(x) \mathrm{J}(x)+(1-\mathrm{t}(x)) \mathrm{A} .
$$

Here, $x$ represents a pixel location, $\mathrm{I}(x)$ is the observed intensity, $\mathrm{J}(x)$ is the scene radiance of a haze-free image, $\mathrm{t}(x)$ is the transmission of light in the atmosphere, inversely related to the scene's depth, and A is the airlight, a global vector quantity describing the ambient light.

When no extra information apart from the input image is considered, the problem is known as single-image dehazing. A common approach is to formulate some restriction on the visual characteristics that a reasonable solution can have, see [2]-[4]. The most popular work is the Dark Channel Prior [5], which states that haze-free images locally have a low value of luminance in some color channel. Some extensions have been further used in [6]-[12]. A second approach is based on machine-learning techniques [10], [13]-[15]. A third group of methods is based on spatially varying image processing techniques. They study the local structure of the image to infer the presence of haze, and try to compensate for this effect, e.g. [16] or [17]. A fusion of white balanced and contrast enhanced versions of the input hazy image has also been proposed [18]. There also exist approaches based on perceptual models, such as Retinex [19]-[21]. One drawback of these methods is that, due to the partial lack of physical information within their formulation, they may present under or overenhanced results, see Fig. 2. In general, a well-designed image dehazing method should enhance visibility in far away areas of the scene, but regions not affected by fog should keep their original luminance and chromatic characteristics.

The main contribution in this paper is a fusion-based variational dehazing method that improves previous works by retaining high contrast and colourfulness enhancing capabilities on far away regions, while preserving image content on nearby regions. To that end, we design a three-step procedure. We first minimize the EVID energy, which was designed for image dehazing [22], but tends to generate some overenhancement on nearby areas. Instead of retaining only the last EVID iterate as the dehazed output, we store images associated to all iterates, corresponding to progressively dehazed images. We then minimize a new energy, that we call $E_{F V I D}$. While the minimum of $\mathrm{E}_{\mathrm{FVID}}$ is a dark image, we can employ its iterates to estimate the degree of degradation of each pixel by computing the difference of saturation (DiffSat) of consecutive iterations. Finally, we combine both sources of information through a fusion procedure that leads to a satisfactorily dehazed output, free of overenhancement artifacts, see Fig. 1 .

\section{VARIATIONAL IMAGE DEHAZING}

To restore haze-degraded outdoor images, authors of [22] proposed to minimize the following image energy:

$$
\begin{aligned}
& \mathrm{E}_{\mathrm{EVID}}\left(\mathrm{I}^{j}\right)=\frac{\alpha}{2} \sum_{x}\left(\mathrm{I}^{j}(x)-\mu^{j}\right)^{2}+\frac{\beta}{2} \sum_{x}\left(\mathrm{I}^{j}(x)-\mathrm{I}_{0}^{j}(x)\right)^{2}- \\
& \frac{\gamma}{2} \sum_{x, y} \omega(x, y)\left|\mathrm{I}^{j}(x)-\mathrm{I}^{j}(y)\right|-\frac{\eta}{2} \sum_{x, y} \omega(x, y)\left|\mathrm{I}^{j}(x)-\mathrm{I}^{j+1}(y)\right| \\
& -\frac{\eta}{2} \sum_{x, y} \omega(x, y)\left|\mathrm{I}^{j}(x)-\mathrm{I}^{j+2}(y)\right|
\end{aligned}
$$

where $\mathrm{I}^{j}$ is a color channel with values in $[0,1], \mathrm{I}_{0}$ is the input, $x, y$ are pixel coordinates, $\alpha, \beta, \gamma, \eta$ are positive parameters, $\omega(x, y)$ is a distance function with values decreasing as the distance between $x$ and $y$ increases, $\mu^{j} \approx 2 \operatorname{mean}\left(\mathrm{I}^{j}\right)-\mathrm{A}^{j}$ is an estimate of the predicted mean of the haze-free image, and $j \in \mathbb{Z}_{3}$. Here, $\{R, G, B\}$ are identified with the space of integers modulo $3, \mathbb{Z}_{3}$, eg $\mathrm{I}^{2}=\mathrm{I}^{G}$, and $\mathrm{I}^{3}=\mathrm{I}^{B}$. This energy is termed Enhanced Variational Image Dehazing (EVID). 


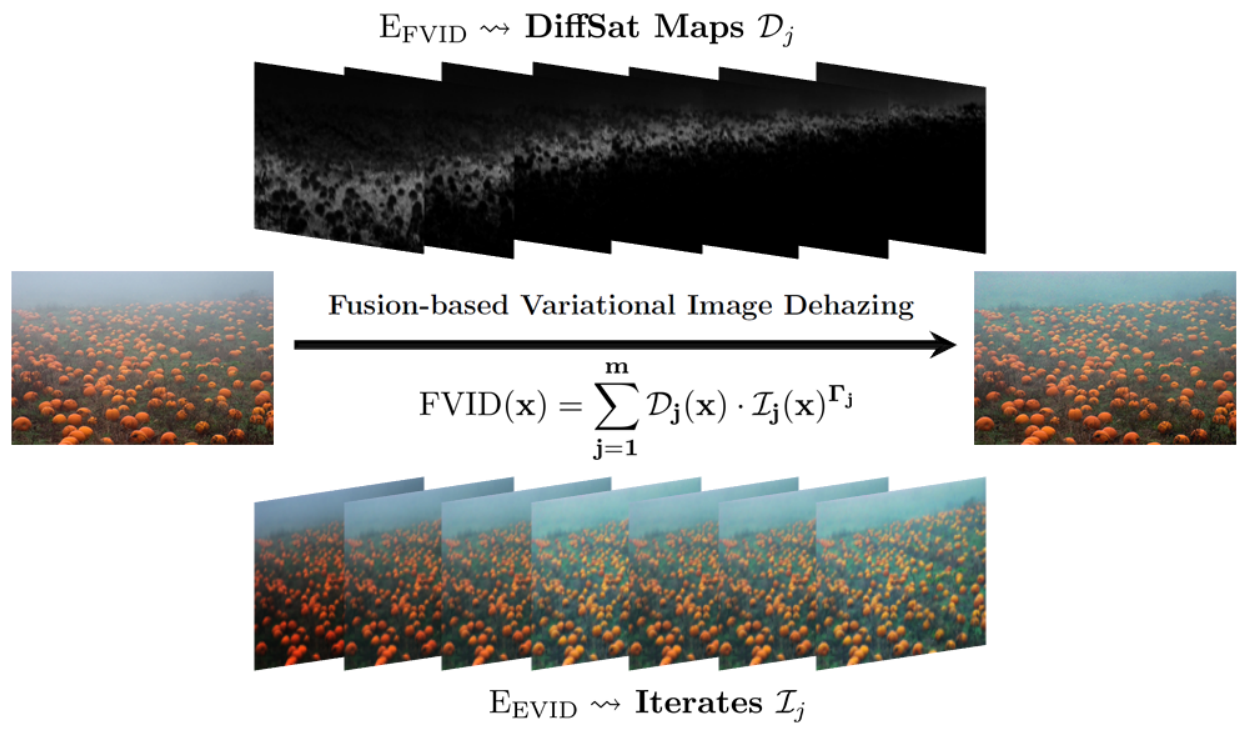

Fig. 1. Schematic representation of the three steps composing the FVID technique.

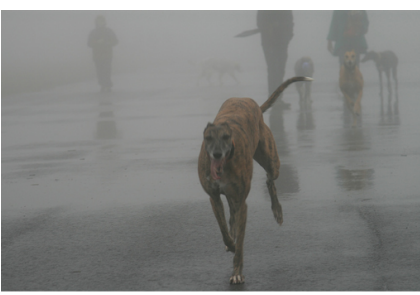

(a)

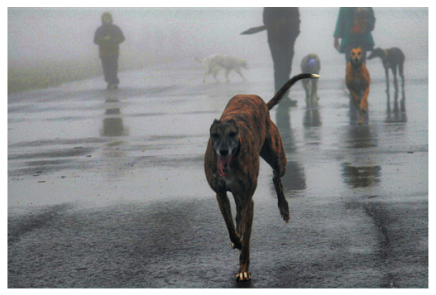

(b)

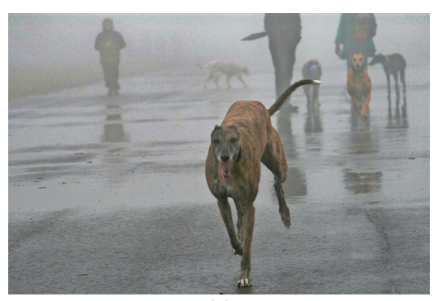

(c)

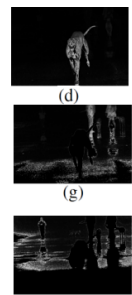

(j)
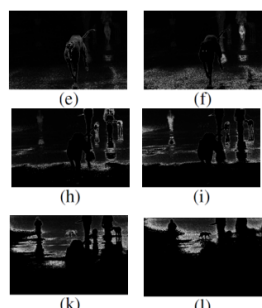

Fig. 2. (a) A hazy scene, and the result of applying (b) EVID dehazing technique (c) The proposed FVID method. (d)-(l) The progressive DiffSat maps generated by our approach. Note the loss of details in the face of the dog produced by EVID. This overenhancement artifact is properly corrected by FVID.

\section{FUSION-BASED VARIATIONAL IMAGE DEHAZING}

Despite the good dehazing capabilities of (2), it sometimes produces overenhanced results, see fig. 2. Nonetheless, each generated iterate implicitly carries useful information on the degree of enhancement each region needs. This observation leads us to design a method to extract such information, in the form of progressive Difference-of-Saturations (DiffSat) maps. After building those maps, we use them to formulate a fusion process to blend the EVID iterates into a single image, keeping only the most useful visual information at each input layer.

\section{A. Progressive DiffSat Maps Generation}

To capture the implicit depth information that EVID generates, we extend the energy of (2) as follows:

$$
\mathrm{E}_{\mathrm{FVID}}\left(\mathrm{I}^{j}\right)=\mathrm{E}_{\mathrm{EVID}}\left(\mathrm{I}^{j}\right)+\tau \sum_{x} \mathrm{I}^{j}(x) .
$$

This straightforward extension keeps the dehazing capabilities of EVID, while it adds a penalty term for large image intensity values. In practice, this amounts to prioritizing a dark image.
To minimize the $\mathrm{E}_{\mathrm{FVID}}$ energy, the Euler-Lagrange equations for $\mathrm{E}_{\mathrm{FVID}}$ can be easily derived (see [22]):

$$
\begin{aligned}
\nabla \mathrm{E}_{\mathrm{FVID}}\left(\mathrm{I}^{j}\right) & =\alpha\left(\mathrm{I}^{j}-\mu_{j}\right)+\beta\left(\mathrm{I}^{j}-\mathrm{I}_{0}^{j}\right)-\gamma R\left(\mathrm{I}^{j}, \mathrm{I}^{j}\right) \\
& -\eta\left(R\left(\mathrm{I}^{j}, \mathrm{I}^{j+1}\right)+R\left(\mathrm{I}^{j}, \mathrm{I}^{j+2}\right)\right)+\tau=0
\end{aligned}
$$

for $j \in\{R, G, B\}$. Here, $R\left(\mathrm{I}^{i}, \mathrm{I}^{j}\right)$ is defined as follows:

$$
R\left(\mathrm{I}^{i}, \mathrm{I}^{j}\right)(x)=\frac{\sum_{y} \omega(x, y) s\left(\mathrm{I}^{i}(x)-\mathrm{I}^{j}(y)\right)}{\sum_{y} \omega(x, y)},
$$

being $s$ a sigmoid function, and with $j$ defined modulo 3 , varying in the $\{R, G, B\}$ components of the input image.

Since (2) is not differentiable, a smooth approximation of the absolute value should be employed. In here, we follow the same approximation as in [22] to obtain (4).

Once the gradient of the energy is computed, we can advance towards one of its minima by using gradient descent:

$$
\begin{aligned}
& \mathrm{I}_{k+1}^{j}=\mathrm{I}_{k}^{j}(1-\Delta t(\alpha+\beta))+\Delta t\left(\alpha \mu^{j}+\beta \mathrm{I}_{0}\right) \\
& +\Delta t\left[\gamma R\left(\mathrm{I}_{k}^{j}, \mathrm{I}_{k}^{j}\right)+\eta\left[R\left(\mathrm{I}_{k}^{j}, \mathrm{I}_{k}^{j+1}\right)+R\left(\mathrm{I}_{k}^{j}, \mathrm{I}_{k}^{j+2}\right)\right]-\tau\right] .
\end{aligned}
$$

The additional term inserted in (3) penalizes large intensity values in the image. Thus, the above gradient descent shrinks intensity values of the input image. The energy minimization takes place in the space of non-negative images. This imposes 
a non-linear constraint that can be handled with different approaches, e.g. the KKT conditions [23]. A simpler approach employed in this work consists of, when an iteration takes a negative value, back-projecting the image into the $[0,1]$ range by clipping.

Note that the minimization of $\mathrm{E}_{\mathrm{FVID}}$ differs from that of $\mathrm{E}_{\text {EVID }}$ in that we introduce a shift in the Gray-World value. To see this, we reformulate (4) as:

$\nabla \mathrm{E}_{\mathrm{FVID}}\left(\mathrm{I}^{j}\right)=\alpha\left(\mathrm{I}^{j}-\left(\mu_{j}-\frac{\tau}{\alpha}\right)\right)+\beta\left(\mathrm{I}^{j}-\mathrm{I}_{0}^{j}\right)$
$-\gamma R\left(\mathrm{I}^{j}, \mathrm{I}^{j}\right)-\eta\left(R\left(\mathrm{I}^{j}, \mathrm{I}^{j+1}+R\left(\mathrm{I}^{j}, \mathrm{I}^{j+2}\right)\right)=0\right.$.

We are not interested in the minimum of (3), but in the rate at which intensity values of individual pixels $\mathrm{I}(x, y)$ tend to zero. Closer regions have more saturated pixels, and their values rapidly decrease driven by the inter-channel contrast maximization. Far-away regions contain mostly achromatic non-saturated pixels, that take more time to vanish. We thus observe the difference in saturation of each iteration with respect to the previous one. Then, we generate a series of Difference-of-Saturations (DiffSat) maps to accurately reflect the depth distribution in the scene:

$$
\mathrm{D}_{k}=\operatorname{Sat}\left(\mathrm{I}_{k}\right)-\operatorname{Sat}\left(\mathrm{I}_{k-1}\right),
$$

where $\mathrm{I}_{k}$ are the iterations from (6), and $\operatorname{Sat}\left(\mathrm{I}_{k}\right)$ is the saturation of $\mathrm{I}_{k}, \operatorname{Sat}\left(\mathrm{I}_{k}\right)=\left(\max _{j}\left(\mathrm{I}_{k}^{j}\right)-\min _{j}\left(\mathrm{I}_{k}^{j}\right)\right) / \max _{j}\left(\mathrm{I}_{k}^{j}\right)$, for $j \in\{R, G, B\}$, and being $\mathrm{I}_{0}=\mathrm{I}$ the input. Figures (1.d)(1.l) display the generated DiffSat maps.

\section{B. Fusion Procedure}

After the minimization of (2) and (3) has generated the EVID iterates $\left\{\mathrm{I}_{j}\right\}_{j=1}^{N}$ and the DiffSat maps $\left\{\mathrm{D}_{k}\right\}_{k=1}^{M}$ respectively, it remains to fuse both information sources. The fusion process should reflect that DiffSat maps corresponding to late iterates, i.e. to those regions with higher fog, correlate with further processed haze-free iterations.

First, we either interpolate or extrapolate the set of DiffSat maps to obtain a new set of exactly $N$ depth maps. These new maps are convolved with a Gaussian kernel in three dimensions $(x, y$, and temporal) in order to present smoothed transitions, and they are later normalized so that the sum in the temporal dimension for any pixel $x$ is equal to 1 . We finally compute the fused image as the combination of these normalized depth maps and the haze-free iterates as:

$$
\operatorname{FVID}(x)=\sum_{j=1}^{N} \mathcal{D}_{j}(x) \cdot \mathcal{I}_{j}(x)^{\Gamma_{j}}
$$

where $\Gamma=\left[\Gamma_{1}, \cdots, \Gamma_{l}\right]$ is a set of increasing values between 0.45 and 1.2 that counter-effect the fact that the original image is in linear form (i.e. it is not gamma corrected).

\section{EXPERIMENTAL EVALUATION}

We implement (2) and (3) with the following reference values: $\alpha=0.5, \beta=0.5, \gamma=0.2, \eta=0.02$ and $\tau=1$. Both distance functions are Gaussians with a standard deviation of
50 pixels. The time step was set to $\Delta t=0.15$ for (2) and $\Delta t=0.05$ for $(3)$, to allow for a more fine-grained DiffSat map set. We consider that a steady-state of the gradient descent is achieved when the difference between the images of two consecutive iterations is below 0.02 . The set of $\Gamma$ values for (9) is equally spaced between 0.45 (value considered for the first iterate) and 1.2 (value employed for the last one). In terms of computational load, FVID doubles the complexity of EVID, since it minimizes both models (2) and (3). We typically observe a number of iterations between 6 and 12. The FFT technique proposed in [24] can also be applied here, delivering an $\mathcal{O}(n)$ complexity, which in our implementation requires 90 seconds to process a 1 MP image ${ }^{1}$ An algorithmic analysis of the minimization of the energy in [24], which lies at the core of the proposed technique, is available at [25].

Fig. (3.a) describes a typical foggy scene. While all methods improve the visibility of the wheat piles, the method in [4] and EVID suffer from heavy overenhancing. Here, the approaches of [3], [6], and FVID seem to recover a well-dehazed result. We confirm it by conducting a simple experiment. First, we obtain a depth map from the Dark Channel method, which provides a reliable transmission map for images degraded by homogeneous fog. We normalize the transmission and threshold it at successive depth levels, to obtain cumulative depth maps and corresponding partial images, see figs. (3.b) to (3.e). For each thresholded depth map, we measure the deviation of the original image from the dehazed results in terms of image structure, applying the SSIM index [26]. We see in (3.g) that every dehazing method produces a decreasing sequence of SSIM scores, as expected. However, the method of [4] and EVID produce rapidly decreasing scores. This implies that they aggressively modify nearby regions when dehazing far-away areas. This is confirmed by the overenhanced results shown on figs. (4.b) and (4.e). Conversely, the methods of [3], [6] and FVID produce more balanced successive SSIM scores, i.e., they better preserve nearby areas. While far-away areas of the scene seem to be satisfactorily dehazed by all the methods, the FVID approach produces a dehazed image that slowly separates from the input hazy scene, dehazing the scene progressively while better keeping the structural similarity in less hazy areas.

Like EVID, FVID dehazes images even when they contain uneven illumination. The sun located on the left of fig. 4 produces a non-uniform illumination. As usual in dehazing methods that assume a regular distribution of haze, the techniques in [3], [5], [15] overcompensate the illumination in the left area, introducing yellowish artificial tones. In contrast, EVID and FVID increase visibility (see the central tree) without introducing color artifacts. EVID produces excessive contrast on nearby regions, as observed in the areas of grass close to the camera. These undesired artifacts are satisfactorily avoided by FVID.

Another seldom addressed problem is the appearance of chromatic artifacts in sky regions [27], [28], Here, we take

\footnotetext{
${ }^{1}$ The software to reproduce the results shown in this paper is available for download at https://sites.google.com/site/agaldran/software
} 


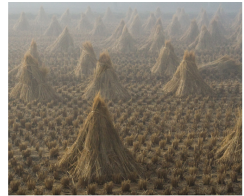

(a)

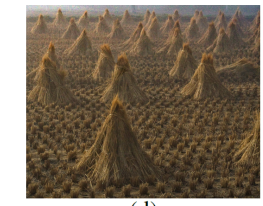

(d)

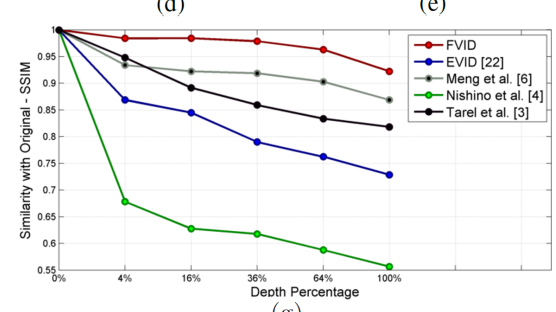

(g)

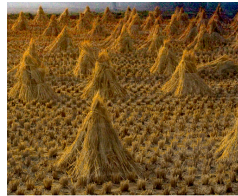

(b)

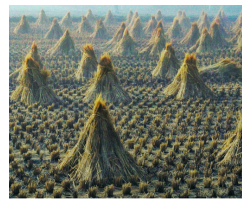

(e)

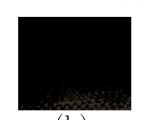

(h)

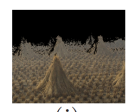

(j)

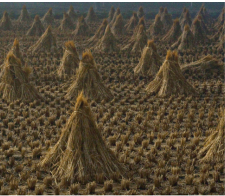

(c)

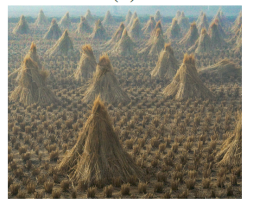

(f)

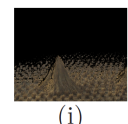

(i)

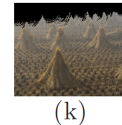

(k)
Fig. 3. Hazy scene and outputs of different dehazing methods: (b) BD [4], (c) NBP [3], (d) BCCRD [6], (e) EVID [22], and (f) FVID. (g) SSIM of partial images produced masking dehazed outputs by binarized depth maps: (h)-(k) show progressive masked images at a depth of: (h) $4 \%$ (i) $16 \%$ (j) $36 \%$ (k) $64 \%$.

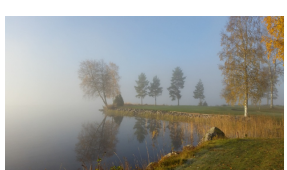

(a)

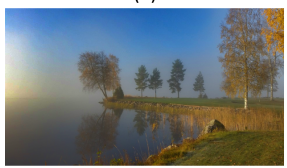

(d)

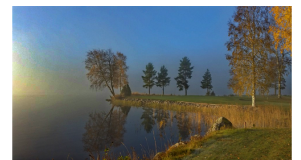

(b)

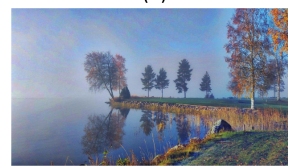

(e)

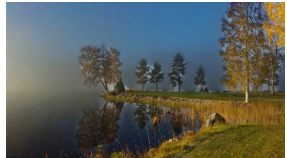

(c)

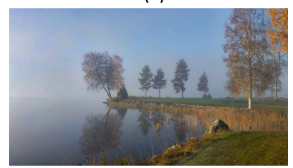

(f)
Fig. 4. A hazy scene with an uneven illumination and the outputs of: (b) FADE [15], (c) No-black pixel [3], (d) Dark channel [5], (e) EVID [22], and (f) FVID.

advantage of the flexibility of the variational approach to process sky areas on the image without introducing overenhancing artifacts. We consider an adaptive weighting strategy for the attachment to data term in the DiffSat map generation scheme: we modify $\beta$ in (3) as an array of increasing weights, $\beta=\left(\beta_{i}\right)=\left(\beta_{0} \cdot 1.1^{i}\right)$. This produces a set of DiffSat maps that do not consider sky regions as being far away, and leads to a more conservative behaviour of the fusion stage therein. We compare this approach in fig. 5 vs the standard FVID, EVID, DC [5] and FADE [15], to show the artifact removal effect of the adaptive attachment-to-data term. The chromatic distortion generated by standard FVID on sky regions is mitigated by this modified version, while contrast gain is retained in other areas, including far-away buildings. We have zoomed in the sky region to better observe these distortions. They are substantially alleviated with the adaptive attachment-to-data strategy.

To quantitatively evaluate FVID, we perform a similar procedure as in [22]. We simulate a dataset composed of 48

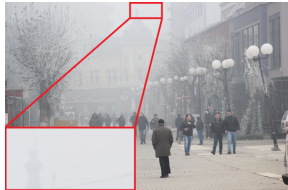

(a)

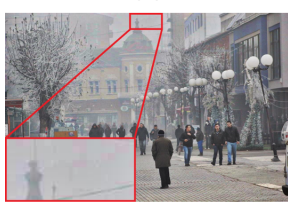

(d)

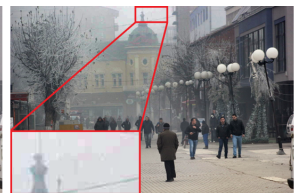

(b)

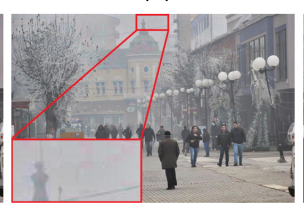

(e)

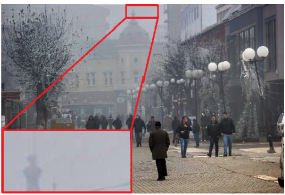

(c)

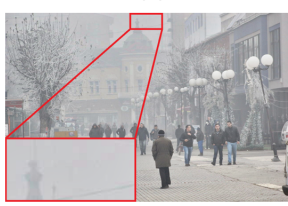

(f)
Fig. 5. Hazy scene with sky region, and results of: (b) DC [5], (c) FADE [15], (d) EVID, (e) FVID, and (f) FVID with adaptive attachment to data.

foggy images: 12 scenes from [29] are degraded with 4 different realistic fog layers, generated with the software provided in [30]. We compare FVID vs. other recent methods, namely EVID [22], and the methods of [5], [3], and [31]. Again, FVID was computed with the same fixed parameters for all scenes.

In this letter, we slightly modify the five image metrics used in [22]. Here, the first measure computes the peaksignal-to-noise ratio (PSNR) between each channel of the images and combines the results of the three channels using the $l_{2}$ norm: $\operatorname{PSNR}_{s p l i t}=\sqrt{\sum_{c=r, g, b} \operatorname{PSNR}\left(I_{c}^{G T}, I_{c}^{m}\right)^{2}}$, where $I^{G T}$ is the ground-truth image, $I^{m}$ is the method used, and the subindexes are each color channel. The second measure is the PSNR between the luminance of the images. The third measure is computed as $l_{2}-$ color $=$ $\sum_{i=1}^{M} \sqrt{\sum_{c=r, g, b}\left(I_{c}^{G T}(i)-I_{c}^{m}(i)\right)^{2}} / M$, where the images are in the range $(0,255)$. The last two correlation measures are defined as $\operatorname{Corr}_{s p l i t}=\sqrt{\sum_{c=r, g, b} \operatorname{Corr}\left(I_{c}^{G T}, I_{c}^{m}\right)^{2}}$, and $\operatorname{Corr}_{\text {lum }}=\operatorname{Corr}\left(I_{\text {lum }}^{G T}, I_{\text {lum }}^{m}\right)$, where Corr gives the correlation coefficient between the images. Results are shown in Table II. For all the evaluated measures, except for the $l 2-$ color, a higher value means a better method. The proposed FVID method outperforms all the others.

TABLE I

QUANTITATIVE RESULTS AS THE MEAN FOR 48 IMAGES

\begin{tabular}{|c|c|c|c|c|c|}
\hline Error measure & {$[\overline{31}]$} & {$[\overline{3}]$} & {$[\overline{5}]$} & EVID $[\overline{22}]$ & FVID \\
\hline 12-color & 47.96 & 52.03 & 48.93 & $47.1 \overline{8}$ & $\mathbf{4 4 . 4 2}$ \\
\hline$P S N R_{\text {lum }}$ & 16.03 & 16.52 & 15.53 & 17.00 & $\mathbf{1 7 . 3 1}$ \\
\hline$P S N R_{\text {split }}$ & 6.90 & 6.99 & 6.79 & 7.13 & $\mathbf{7 . 1 9}$ \\
\hline Corr $_{\text {split }}$ & 1.24 & 1.21 & 1.18 & 1.25 & $\mathbf{1 . 2 6}$ \\
\hline Corr $_{\text {lum }}$ & 0.70 & 0.69 & 0.66 & 0.71 & $\mathbf{0 . 7 2}$ \\
\hline
\end{tabular}

\section{Discussion AND CONCLUSIONS}

We have described FVID, a novel haze removal method. It extends significantly upon a previously proposed variational dehazing scheme (EVID) by integrating information from its iterates and a hierarchical set of DiffSat maps coming from an extension of the EVID image energy. A fusion of visual information coming from both sources leads to an effective 
dehazing technique. FVID achieves results comparable to stateof-the-art techniques when enhancing far-away regions, while it preserves nearby regions. We propose also a variant of FVID to handle overenhancement artifacts that appear when dehazing scenes with sky regions. Moreover, the flexibility of variational methods allows to generate families of FVID methods with different features, which shall be explored in future work.

\section{REFERENCES}

[1] H. Koschmieder, Theorie der horizontalen Sichtweite: Kontrast und Sichtweite. Keim \& Nemnich, 1925.

[2] R. Fattal, "Single Image Dehazing," in ACM SIGGRAPH 2008 Papers, ser. SIGGRAPH '08. New York, NY, USA: ACM, 2008, pp. 72:1-72:9.

[3] J. P. Tarel and N. Hautiere, "Fast visibility restoration from a single color or gray level image," in 2009 IEEE 12th International Conference on Computer Vision (ICCV), Sep. 2009, pp. 2201-2208.

[4] K. Nishino, L. Kratz, and S. Lombardi, "Bayesian Defogging," Inter national Journal of Computer Vision, vol. 98, no. 3, pp. 263-278, Jul. 2012.

[5] K. He, J. Sun, and X. Tang, "Single Image Haze Removal Using Dark Channel Prior," IEEE Transactions on Pattern Analysis and Machine Intelligence, vol. 33, no. 12, pp. 2341-2353, Dec. 2011.

[6] G. Meng, Y. Wang, J. Duan, S. Xiang, and C. Pan, "Efficient Image Dehazing with Boundary Constraint and Contextual Regularization," in 2013 IEEE International Conference on Computer Vision (ICCV), Dec. 2013, pp. 617-624.

[7] W. Sun, "A new single-image fog removal algorithm based on physical model," Optik - International Journal for Light and Electron Optics, vol. 124, no. 21, pp. 4770-4775, Nov. 2013.

[8] Y. Gao, H.-M. Hu, S. Wang, and B. Li, "A fast image dehazing algorithm based on negative correction," Signal Processing, vol. 103, pp. 380-398, Oct. 2014

[9] J.-B. Wang, N. He, L.-L. Zhang, and K. Lu, "Single image dehazing with a physical model and dark channel prior," Neurocomputing, vol 149, Part B, pp. 718-728, Feb. 2015

[10] Q. Zhu, J. Mai, and L. Shao, "A Fast Single Image Haze Removal Algorithm Using Color Attenuation Prior," IEEE Transactions on Image Processing, vol. 24, no. 11, pp. 3522-3533, Nov. 2015.

[11] Z. Li and J. Zheng, "Edge-Preserving Decomposition-Based Single Image Haze Removal,' IEEE Transactions on Image Processing, vol. 24, no. 12 , pp. 5432-5441, Dec. 2015.

[12] Y.-H. Lai, Y.-L. Chen, C.-J. Chiou, and C.-T. Hsu, "Single-Image Dehazing via Optimal Transmission Map Under Scene Priors," IEEE Transactions on Circuits and Systems for Video Technology, vol. 25, no. 1, pp. 1-14, Jan. 2015.

[13] K. Tang, J. Yang, and J. Wang, "Investigating Haze-Relevant Features in a Learning Framework for Image Dehazing," in 2014 IEEE Conference on Computer Vision and Pattern Recognition (CVPR), Jun. 2014, pp. 2995-3002.

[14] B. Cai, X. Xu, K. Jia, C. Qing, and D. Tao, "DehazeNet: An End-to-End System for Single Image Haze Removal," IEEE Transactions on Image Processing, vol. 25, no. 11, pp. 5187-5198, Nov. 2016.

[15] L. K. Choi, J. You, and A. C. Bovik, "Referenceless Prediction of Perceptual Fog Density and Perceptual Image Defogging," IEEE Transactions on Image Processing, vol. 24, no. 11, pp. 3888-3901, Nov. 2015.

[16] R. Tan, "Visibility in bad weather from a single image," in 2008 IEEE Conference on Computer Vision and Pattern Recognition (CVPR), Jun. 2008, pp. 1-8.

[17] J. Oakley and H. Bu, "Correction of Simple Contrast Loss in Color Images," IEEE Transactions on Image Processing, vol. 16, no. 2, pp. 511-522, Feb. 2007.

[18] C. Ancuti and C. Ancuti, "Single Image Dehazing by Multi-Scale Fusion," IEEE Transactions on Image Processing, vol. 22, no. 8, pp. 3271-3282, Aug. 2013.

[19] V. De Dravo and J. Hardeberg, "Stress for dehazing," in Colour and Visual Computing Symposium (CVCS), 2015, Aug. 2015, pp. 1-6.

[20] X.-S. Zhang, S.-B. Gao, C.-Y. Li, and Y.-J. Li, "A Retina Inspired Model for Enhancing Visibility of Hazy Images," Frontiers in Computational Neuroscience, p. 151, 2015.
[21] Y. Wang, H. Wang, C. Yin, and M. Dai, "Biologically inspired image enhancement based on Retinex," Neurocomputing, vol. 177, pp. 373-384, Feb. 2016.

[22] A. Galdran, J. Vazquez-Corral, D. Pardo, and M. Bertalmío, "Enhanced Variational Image Dehazing," SIAM Journal on Imaging Sciences, vol. 8, no. 3, pp. 1519-1546, Jan. 2015

[23] J.-B. Hiriart-Urruty and C. Lemaréchal, Convex Analysis and Minimization Algorithms I, M. Artin, S. S. Chern, J. Coates, J. M. Fröhlich, H. Hironaka, F. Hirzebruch, L. Hörmander, C. C. Moore, J. K. Moser, M. Nagata, W. Schmidt, D. S. Scott, Y. G. Sinai, J. Tits, M. Waldschmidt, S. Watanabe, M. Berger, B. Eckmann, and S. R. S. Varadhan, Eds. Berlin, Heidelberg: Springer Berlin Heidelberg, 1993.

[24] M. Bertalmío, V. Caselles, E. Provenzi, and A. Rizzi, "Perceptual color correction through variational techniques," IEEE Transactions on Image Processing, vol. 16, no. 4, pp. 1058-1072, Apr. 2007.

[25] S. Ferradans, R. Palma-Amestoy, and E. Provenzi, "An Algorithmic Analysis of Variational Models for Perceptual Local Contrast Enhancement,' Image Processing On Line, vol. 5, pp. 219-233, 2015.

[26] Z. Wang, A. Bovik, H. Sheikh, and E. Simoncelli, "Image quality assessment: From error visibility to structural similarity," IEEE Transactions on Image Processing, vol. 13, no. 4, pp. 600-612, Apr. 2004

[27] Y.-b. Zhu, J.-m. Liu, and Y.-g. Hao, "A single image dehazing algorithm using sky detection and segmentation," in 2014 7th International Congress on Image and Signal Processing (CISP), Oct. 2014, pp. 248-252.

[28] Y. Li, F. Guo, R. T. Tan, and M. S. Brown, "A Contrast Enhancement Framework with JPEG Artifacts Suppression," in Computer Vision -ECCV 2014, ser. Lecture Notes in Computer Science, D. Fleet, T. Pajdla, B. Schiele, and T. Tuytelaars, Eds. Springer International Publishing, Sep. 2014, no. 8690, pp. 174-188.

[29] C.-C. Su, L. Cormack, and A. Bovik, "Color and Depth Priors in Natural Images," IEEE Transactions on Image Processing, vol. 22, no. 6, pp. 2259-2274, Jun. 2013

[30] J.-P. Tarel, N. Hautiere, L. Caraffa, A. Cord, H. Halmaoui, and D. Gruyer, "Vision Enhancement in Homogeneous and Heterogeneous Fog," IEEE Intelligent Transportation Systems Magazine, vol. 4, no. 2, pp. 6-20, Apr. 2012.

[31] K. Gibson and T. Nguyen, "Fast single image fog removal using the adaptive Wiener filter," in 2013 20th IEEE International Conference on Image Processing (ICIP), Sep. 2013, pp. 714-718. 